\title{
Alopecia areata: Assoziation mit Zinkmangel bestätigt
}

\author{
Bei Patienten mit Alopecia areata sind die Zinkspiegel im Serum signifikant verringert. Zudem \\ korrelieren sie invers mit der Therapieresistenz. Und: Je niedriger die Serumspiegel sind, umso \\ ausgeprägter ist die Alopezie, wie die Ergebnisse einer Studie ägyptischer Dermatologen bestätigen.
}

In ihre Fall-Kontroll-Studie nahmen die Dermatologen um Dr. Nermeen S. A. Abdel Fattah von der Universität Kairo insgesamt 100 Teilnehmer randomisiert auf, von denen die eine Hälfe Gesunde der Kontrollgruppe waren. Von den 50 Patienten war bei 25 die Autoimmunerkrankung innerhalb des vergangenen halben Jahres aufgetreten. Bei den übrigen 25 Patienten dagegen bestand die Erkrankung bereits seit mindestens 6 Monaten, und die Patienten hatten zudem auf 3 oder mehr Therapien nicht angesprochen. Die Alopezie war jeweils unterschiedlich stark ausgeprägt, von nur einem einzigem Herd bis zur Alopecia totalis. Das Ausmaß der Läsionen wurde mithilfe des SALT(severity of alopecia tool)-Scores erfasst: von S0 (kein Haarverlust) bis S5 (100\% Haarverlust) auf der Kopfhaut, sowie B0 bis B3 (kein Haarverlust bis $100 \%$ Haarverlust) am übrigen Körper.

\section{Messung des Spurenelements im Nüichternblut}

Die Messung des essenziellen Spurenelements erfolgte jeweils einmal, und zwar im Serum nach Entnahme von venösem Nüchternblut. Vor der Messung der Zinkkonzentration war das Serum bei $-20^{\circ} \mathrm{C}$ in Polypropylenröhrchen eingefroren worden. Die Menge an Zink im Serum wurde mithilfe der GraphitrohrAtomabsorptionsspektroskopie bestimmt. Als Referenzwert gilt eine Zinkkonzentration von 60 bis $110 \mu \mathrm{g} / \mathrm{dl}$.

Im Vergleich zur Kontrollgruppe mit einer Konzentration von $85,7 \pm 12,50 \mu \mathrm{g} / \mathrm{dl}$ waren die Werte bei den Alopeziepatienten deutlich niedriger $(75,48 \pm 11,78 \mu \mathrm{g} / \mathrm{dl})$. Besonders groß war der Unterschied zur Subgruppe der Patienten mit Therapieresistenz $(71,44 \pm 9,87 \mu \mathrm{g} / \mathrm{dl})$, deren Zinkspiegel zudem signifikant niedriger waren als die der Patienten mit erst kürzlich diagnostizierter Erkrankung (79,52 $\pm 12,33 \mu \mathrm{g} / \mathrm{dl})$.

Nach Angaben der Dermatologen gab es darüber hinaus eine signifikante inverse Korrelation zwischen der Zinkkonzentration und dem Ausmaß der Läsionen ( $r=-0,573 ; \mathrm{p}=0,001)$ : Je niedriger die Zinkspiegel im Serum waren, umso größer war der SALT-Score. Am Stärksten ausgeprägt war die Korrelation bei therapieresistenter Erkrankung $(r=-0,655 ; p=0,001)$.
Auch beim Zusammenhang zwischen Zinkspiegel und Dauer der Alopezie entdeckten die Ärzte über die gesamte Studiengruppe hinweg eine inverse Korrelation $(r=-0,530 ; \mathrm{p}=0,001)$. Signifikant war dieser Zusammenhang nur in der Gruppe der Patienten mit Therapieresistenz $(r=-0,965 ; \mathrm{p}=0,001)$.

Aus den Ergebnissen leiten die Studienautoren ab, dass niedrige Zinkspiegel ursächlich im Zusammenhang mit der Therapieresistenz stehen, da die Zinkkonzentration bei Patienten mit neu diagnostizierter Erkrankung signifikant höher liegt, wenn auch nicht im Referenzbereich. Sie halten den Zinkspiegel für einen geeigneten Marker für das Ausmaß und die Dauer der Alopezie. Die Patienten könnten möglicherweise von einer Supplementierung profitieren.

Als pathogenetischen Zusammenhang zwischen Zinkmangel und Alopecia areata vermuten Wissenschaftler unter anderem eine Einschränkung der T-Zell-Funktion. Darüber hinaus verlangsamt die Unterversorgung der Zellen mit Zink das Wachstum der Haare, die außerdem leichter abbrechen.

Peter Leiner

Abdel Fattah NS et al. Int J Dermatol 2015, Jul 3; doi:10.1111/ijd.12769 